\title{
Imaging stress
}

\author{
Shlomi Brielle ${ }^{1,2} \cdot$ Rotem Gura $^{3} \cdot$ Daniel Kaganovich $^{1}$
}

Received: 11 February 2015 / Revised: 16 June 2015 / Accepted: 17 June 2015 / Published online: 4 July 2015

(C) Cell Stress Society International 2015

\begin{abstract}
Recent innovations in cell biology and imaging approaches are changing the way we study cellular stress, protein misfolding, and aggregation. Studies have begun to show that stress responses are even more variegated and dynamic than previously thought, encompassing nano-scale reorganization of cytosolic machinery that occurs almost instantaneously, much faster than transcriptional responses. Moreover, protein and mRNA quality control is often organized into highly dynamic macromolecular assemblies, or dynamic droplets, which could easily be mistaken for dysfunctional "aggregates," but which are, in fact, regulated functional compartments. The nano-scale architecture of stressresponse ranges from diffraction-limited structures like stress granules, P-bodies, and stress foci to slightly larger quality control inclusions like juxta nuclear quality control compartment (JUNQ) and insoluble protein deposit compartment (IPOD), as well as others. Examining the biochemical and physical properties of these dynamic structures necessitates live cell imaging at high spatial and temporal resolution, and techniques to make quantitative measurements with respect to
\end{abstract}

Electronic supplementary material The online version of this article (doi:10.1007/s12192-015-0615-y) contains supplementary material, which is available to authorized users.

Daniel Kaganovich

dan@cc.huji.ac.il

1 Department of Cell and Developmental Biology, Alexander Silberman Institute of Life Sciences, Hebrew University of Jerusalem, Jerusalem 91904, Israel

2 Alexander Grass Center for Bioengineering, Hebrew University of Jerusalem, Jerusalem, Israel 91904

3 Department of Physics, Massachusetts Institute of Technology, Cambridge, MA 02139, USA movement, localization, and mobility. Hence, it is important to note some of the most recent observations, while casting an eye towards new imaging approaches that offer the possibility of collecting entirely new kinds of data from living cells.

Keywords Chaperone $\cdot$ Misfolded protein $\cdot$ Aggregation FRAP · FLIP · PhoC · Inclusion · JUNQ · IPOD · Stress foci · Stress granules $\cdot$ P-bodies $\cdot$ Dynamic droplets

\section{Stress}

Protecting the cell from protein-associated damage is a matter of having the correct proteins at the right place at the right time: the cellular environment changes rapidly in different folding and stress conditions in order to avoid catastrophic consequences of too many misfolded polypeptides or not enough functional proteins. When a cell is exposed to stresses such as heat-shock, cold-shock, osmotic stress, starvation, or amino-acid analogues which cause rampant mutations, the cellular response propagates across the entire network of protein biogenesis.

Although much research has focused on how these stresses affect protein synthesis, we are only now beginning to look closely at how stress effects protein localization and distribution. Whereas expression takes time, and is more difficult to accomplish under stress, protein localization is substantially more dynamic and therefore changes in protein distribution can be affected quickly, in time to deal with the stress. Hence, regulation of protein distribution deserves careful attention in the study of stress response. Indeed, recent work has shown that in the single cell eukaryote Saccharomyces cerevisiae more than half of the proteome radically changes its localization under different stress condition (Breker et al. 2013). By altering the spatial positioning of proteins, cells can change 
their metabolic response by shifting resources to accommodate new conditions. Spatial changes can be affected by shifting proteins between organelles or by compartmentalizing them into inclusions, thereby changing their local concentrations.

Many recent studies underscore the role of spatial architecture to effective stress response. Following acute stress, translation is blocked or stalled and mRNAs (likely together with translation machinery) are routed to storage in stress granules (SGs) (Anderson and Kedersha 2002). Misfolded proteins are collected in similar structures, called stress foci (SFs) together with chaperones (Amen and Kaganovich 2014; Spokoini et al. 2012; Malinovska et al. 2012; Moldavski et al. 2015; EscusaToret et al. 2013). In certain cases, especially under nutrient restrictive conditions, many proteins undergo amyloid-like reversible polymerization in fibrils, which form and disassemble as a function of the cellular $\mathrm{pH}$, and appear to act as storage compartments for biosynthetic machinery in times where it is not used (Alberti 2012).

If stress persists for longer, stress response is once again reconfigured to adapt its function to a changing environment. SGs are converted into P-bodies where mRNAs are de-capped and degraded (Kedersha et al. 2005) and SFs are converted into a juxta nuclear quality control compartment (JUNQ) and an insoluble protein deposit compartment (IPOD) inclusions where misfolded proteins are either eliminated in the JUNQ or become insoluble aggregates in the IPOD (Amen and Kaganovich 2014; Spokoini et al. 2012; Kaganovich et al. 2008; Gallina et al. 2015). Inclusion structures are not static, and can change their composition, mobility properties, and exchange rates depending on the level and nature of stress (Weisberg et al. 2012; Pattabiraman and Kaganovich 2014). Although most of the evidence regarding the biogenesis, function, and properties of JUNQ, IPOD, and SFs come from work done in yeast, there are recent studies suggesting a relatively high degree of conservation for some of these inclusions in higher eukaryotes (Kaganovich et al. 2008; Weisberg et al. 2012; Ogrodnik et al. 2014; Moldavski et al. 2015). For example, a recently published study demonstrated the direct association between inclusions structures and lipid droplets in yeast and mammalian cells (Moldavski et al. 2015).

\section{Dynamic droplets}

In recent years, it has become apparent that membraneless macromolecular assemblies (or dynamic droplets as we call them) are a highly abundant feature of nano-scale cellular organization and as such are also involved in mechanisms of stress response. These droplets (or inclusions) are typically comprised of proteins containing unstructured domains (such as polyglutamine stretches), sometimes together with RNA (Anderson and Kedersha 2006; Buchan and Parker 2009; Hyman et al. 2014). Examples include ribonucleoprotein
(RNP) granules, such as germ granules (Eddy 1976; Anderson and Kedersha 2006), stress granules (Anderson and Kedersha 2002; Buchan and Parker 2009), P-bodies (Bashkirov et al. 1997), JUNQ, stress foci (Amen and Kaganovich 2014; Spokoini et al. 2012; Kaganovich et al. 2008; Brock et al. 2015), and cajal bodies (Gall 2003), as well as centrosomes (Mahen and Venkitaraman 2012) and nucleoli (Boisvert et al. 2007). Inclusions maintain their bio-chemical activity by partitioning a dense sub-compartment from the cytoplasm or nucleoplasm comprised of a few types of biomolecules. These inclusion structures are highly dynamic and chemically active; they are created or disbanded across very short time intervals, with molecules constantly transitioning back and forth between the soluble state in the cytoplasm to the droplet state (Brangwynne et al. 2009; Weber and Brangwynne 2012). Droplets can associate with themselves as well as with other dynamic droplet proteins through multivalent promiscuous interactions, forming a liquid-like phase (Brangwynne et al. 2009; Hyman et al. 2014). Phase separation adds an additional level of cellular regulation, since droplets can assemble or disassemble in response to a slight increase or decrease in the concentrations of constituent proteins.

Cytoplasmic dynamic droplets are of great interest for several reasons. First, their properties appear to change in direct response to cellular conditions, in particular the availability of energy in the form of ATP. The proteins which make up dynamic droplets and inclusions are able to exist in an unbound and soluble state. However, when the ATP needed for continuously solubilizing these proteins decreases in its abundance due to stress or aging, the dynamic droplets can transition to a solid-like phase and become much less dynamic and less functional (Nguyen and Bensaude 1994).

Indeed, inclusions formed by misfolded or aggregation prone proteins draw a compelling parallel with cell biological structures that are apparently similar to those observed in the brains of individuals with neurodegenerative disorders (Ross et al. 2004; Ross and Poirier 2005; Ballatore et al. 2007; Knowles et al. 2014). Post-mortem immunohistochemistry offers an end-stage snap-shot of the cell biology of the disease, which usually onset decades prior to examination. Because of the long process of severe degeneration, it is not entirely clear whether post-mortem inclusions represent a bona fide feature of the disease biology, or are a bi-product of wide-spread cell death and dysfunction. Hence, intracellular structures formed by misfolded proteins in model systems may offer valuable insight into the initial subtle and dynamic processes that may trigger disease. Similarly, there is mounting evidence that stress granule and P-body biology is at the cross-roads of several types of genetically mapped forms of amyotrophic lateral sclerosis (ALS). Several different genetic markers for ALS have been identified, and the majority of them are in proteins that function in RNA quality control, localize to 
SGs, or interact with SG proteins (Couthouis et al. 2011; Li et al. 2013; Jucker and Walker 2013). It is now recognized that most forms of ALS, whether with a known genetic marker or "sporadic," contain aggregates of TDP43, which is an SGconstituent protein (Lagier-Tourenne and Cleveland 2009; Udan and Baloh 2011; King et al. 2012). Although the role of HSPs in SG formation and clearance is still poorly understood, several recent studies have demonstrated an association of chaperones such as Cdc48 and sHSPs with SGs (Buchan et al. 2013; Cherkasov et al. 2013). Although the circumstantial pathological evidence for involvement of inclusion body structures and SGs in neurodegenerative disease is overwhelming, the etiological basis of the pathology is as obscure as ever. Hence, it is essential to extend the technical and conceptual toolkit that will bring biochemical questions into living cells enabling researchers to investigate the properties and function of nano-scale macromolecular assemblies.

\section{Phase separation in dynamic droplet formation}

Inclusion formation can be described as a form of phase separation, similar to water vapor forming water droplets. The cytoplasm can undergo de-mixing from a homogenous state into compartmentalized droplets, whose properties may differ from the surrounding cytoplasmic environment (Zhou et al. 2008; Brangwynne et al. 2009; Hyman et al. 2014). Photobleaching studies of $\mathrm{P}$ granules in $C$. elegans have shown that these inclusions demonstrate internal rearrangement and external exchange with the cytoplasm (Brangwynne et al. 2009). Additionally, P granules have been shown to deform under shear flow in a way that characterizes liquids. P granules also form spherical shape inclusions; a spherical shape may indicate minimization of surface area due to surface tension. These characteristics are indicative of a fluid. It is therefore important to study the formation and properties of inclusions in the context of phase transitions (Brangwynne et al. 2009; Hyman et al. 2014). This is especially true in the context of protein aggregation in inclusions. When examining self-association of misfolded proteins in live cells, we tend to think of solid-state structures such as amyloid inclusions (England and Kaganovich 2011), when in fact there is mounting evidence that many inclusions constitute a liquid state (Weisberg et al. 2012; Ogrodnik et al. 2014).

How do dynamic droplets form? The induction of their assembly appears to be stimulated by a nucleation step that can either be the result of diffusing bio-molecules that selfassemble to create an aggregate-like structure or from a template that primes polymerization (Malinovska et al. 2013; Han et al. 2012; Li et al. 2012). Inclusions formed through aggregation usually require higher concentrations of the aggregating bio-molecule, and may only form under specific conditions. In recent work, RNA has been shown to act as a standalone scaffold in forming RNP bodies (Shevtsov and Dundr 2011). Induced inclusions, on the other hand, require external stimuli or specialized structures to form, such as the formation of actin nucleates by the arp $2 / 3$ complex (Malinovska et al. 2013). Other examples include the formation of a nucleolus using ribosomal RNA complexes (Grob et al. 2014) and the centriole formed by centrosomes (Gönczy 2012; Zwicker et al. 2014).

What determines individual droplet size versus the number of inclusions within a cell? Although it is not yet clear how changes in inclusion size affect biological function, size control may be important for the biochemical activity (Brangwynne 2013; Luby-Phelps 1999; Goehring and Hyman 2012; Ellis 2001; van den Berg et al. 1999), especially in the context of stress response. If droplets are too large this may lead to insoluble aggregation. On the other hand, if too few inclusions form or if inclusions are too small, they may not support the necessary biochemical activity needed to adapt to stress conditions. Recent studies have shown that droplet size scales according to its container volume; RNP droplet size is scales to the cell's volume (Singer and Gall 2011), centrosomes, which are important for spindle assembly, go through size scaling in relation to the cells size in C. elegans embryos (Decker et al. 2011). Size scaling is due to resource limitations, which correlate to the volume containing the inclusion. Inclusion size is not only affected by the cellular concentration of the aggregating molecule (Li et al. 2012; Kato et al. 2012), but also by the energetic favorability of inclusion formation (Hyman et al. 2014). For example, aggregation is affected by Laplace pressure caused by surface tension, which pushes for the formation of larger inclusions rather than a larger number of small inclusions. Additionally, other solutes found in the inclusion may also have an effect on inclusion size (Webster and Cates 1998). These phenomena similarly occur in soap bubbles and oil droplets in water, and should be considered when observing inclusions in order to correctly understand inclusion dynamics.

\section{Imaging: basics}

In order to effectively study the dynamic architecture of acute stress response and droplet formation, proteins must be observed at sub-cellular resolution, and as close to singlemolecule resolution as possible. It is difficult to observe proteins under the microscope, because they are very small and they move very fast. The difficulty arises from three main challenges: limitations on spatiotemporal resolution, noisy data, and photo-toxicity. The current imaging technology cannot overcome all these challenges at once, but it enables better coping with certain challenges at the expense of other challenges.

The first consideration for imaging proteins in the cell pertains to spatial and temporal resolution. The ideal imaging 
system would resolve proteins in the nanometer scale and track their motion in less than a millisecond resolution. However, the diffraction limit and the acquisition rates of current imaging systems make it hard to achieve this spatiotemporal resolution. In super resolution techniques, spatial resolution can be gained at the expense of temporal resolution. In these techniques, an image is collected through integration of a series of illuminations at very low intensity, or through a grid that is super-imposed on the image. In each illumination, only a few fluorophores are excited. Thus, the emission pattern of each fluorophore may be fitted to a point spread function of a point source, and the location of the fluorophore may be determined with a greater precision than that possible with standard techniques. However, the merging of a few images reduces the effective acquisition rate of the data.

Another crucial consideration is the signal to noise ratio (SNR). A high SNR is required to reliably quantify the spatial distribution of proteins to a high precision. However, in rapid imaging, it is challenging to obtain a good SNR due to the decrease in signal to shot noise ratio, which results from the stochastic nature of photon emissions by fluorophores. The fewer photons detected, the greater the shot noise affects the measurements. Changing the acquisition rate inevitably reduces the number of detected photons per pixel. Nevertheless, rapid imaging can be done with a good SNR if spatial resolution is expendable. Averaging over neighboring pixels ("binning the data") within each image would decrease shot noise. However, averaging over pixels discards the information on the variation of the fluorophore concentration within these pixels.

The last consideration to mention here is that imaging with high intensity illumination harms living cells. To minimize photo-toxicity, all measurements must be done at a low excitation power. This constraint limits the increase of the SNR by increasing the excitation power.

Taking these considerations together, what does an ideal system for imaging stress look like? A confocal system is a good starting point, since the $z$ sectioning enabled by confocality increases $x y$ resolution. Moreover, since cells are 3D, 3D tracking over time, or 4D imaging, is critical for following rapidly moving structures like SGs that may localize with one protein $1 \mathrm{~min}$ and another protein the next. A resonant scanning system offers a much more rapid frame rate than regular galvano scanners (200-10 vs. $2-0.25 \mathrm{fps})$, and a Piezo stage will enable rapid $z$ sectioning. All of this increases the speed of acquisition and hence decreases photo-toxicity. The ability to detect fluorescence as efficiently as possible is key as well. Usually a $\times 60$ oil objective with high numerical aperture will provide the greatest resolution to brightness compromise in regions close to the coverslip (up to $5 \mu \mathrm{m}$ away) and $\times 60$ water objectives will provide the greatest amount of resolution and brightness beyond that depth into an aqueous samples (e.g., a living cell). Additionally, today many systems can be configured with GaAsP photo-multiplier tube (PMT) detectors, which are more photosensitive than regular PMTs (45\% photon efficiency vs. $22 \%$ ).

An alternative imaging system may prove advantageous over the confocal system in enhancing temporal resolution and reducing photo-toxicity even further. In single-plane illumination microscopy (SPIM) (sometimes also called light sheet microscopy), instead of illuminating the sample in the direction parallel to the optical axis of the objective, the incident laser beam is focused to a narrow light sheet that is perpendicular to the optical axis. SPIM was developed in 2004 to overcome the difficulty of imaging thick sample using confocal microscopy, which has a limited penetration depth (Huisken et al. 2004; Santi 2011). Illuminating the sample from the side helped in overcoming this obstacle. It also improved the capabilities of imaging 3D samples: since fluorophores outside of the light sheet are rarely excited, they rarely get bleached. Thus, compared to a laser-scanning confocal microscope (LSCM), fewer excitations are needed to acquire a 3D image stack of the same quality. Finally, SPIM enables a faster acquisition rate than LSCM, since no scanning is involved in acquisition. Thus, the entire frame can be collected with a camera such as an electron multiplying charge coupled device (EMCCD) or complementary metal-oxide semiconductor (CMOS). Acquisition by a camera also allows for higher sensitivity of detection since most CCDs are more photon efficient 60-90 \% than PMTs (20-45\%).

Finally, an additional way to reduce photo-toxicity is to use red or far red fluorophores wherever possible, since imaging in far red wavelengths causes less phototoxic stress to the cell. Although in the past, there have been fewer far red than red and green fluorophores in the cell biologists tool kit, excellent far red fluorophores have been developed recently. iRFP (Filonov et al. 2011) in particular is extremely bright and truly far red (emitting at a wavelength close to $700 \mathrm{~nm}$ ) . Additionally, Orange2 (Shaner et al. 2008; Kremers et al. 2009) is a red fluorophore which can be photo-converted to far red with a 488-nm laser pulse.

\section{Dynamic imaging}

Most cell biological structures are highly dynamic, whereas stress-induced cytosolic structures like stress granules, stress foci, and inclusions are much more so. Hence, laser manipulation techniques such as fluorescence loss in photobleaching (FLIP) and fluorescence recovery after photobleaching (FRAP) have long been considered indispensable to studying protein dynamics and structural properties of the cytosol. These techniques are beginning to be eclipsed by much more subtle and quantitative approaches. In particular, photoconversion of fluorophores (PhoC) can be used to generate much more relevant data than FLIP or FRAP, without the artifacts inherent in those approaches and with minimal 
cellular perturbation. There are several disadvantages to traditional FRAP and FLIP approaches in live cells imaged on a typical confocal system. First of all, to bleach the required amount of fluorescence for FRAP (ideally around $75 \%$ ), it is necessary to illuminate the cells with high amounts of 488$\mathrm{nm}$ laser light. Moreover, while FLIP assumes that the bleached region is contained within the region of interest (ROI), the laser beam usually hits a bigger volume than the ROI. Thus, it is not only the ROI fluorescence that is being depleted, but also quite a bit of soluble cytosolic fluorescence, from which the FRAP recovery is supposed to occur. When working in small cells (such as yeast for example) doing FRAP on diffraction-limited structures is simply not realistic, and difficult to quantitate. FLIP has a similar problem - it is supposed to work by continuously depleting fluorescence in the soluble cytosolic fraction of the cell, measuring the rate of exchange in a specific structure or compartment. This causes very high levels of illumination and phototoxic damage to the cell, and is also plagued by the artefactual problem of high levels of illumination outside of the ROI. Hence, even completely immobile structures will eventually decrease in fluorescence in a FLIP experiment.

An ideal alternative to FRAP and FLIP is provided by photoconvertible fluorophores such as Dendra2, Eos3.2, and mOrange2. Dendra2 (Fig. 1), in particular, is rapidly and irreversibly converted from green to red emission with a short low-power laser pulse with a 405-nm laser. Since the starting amount and the post-PhoC amount is both recorded in the imaging (a dual-scanning system with two laser paths for acquisition and stimulation can image and $\mathrm{PhoC}$ simultaneously), PhoC will generate the same type of data from a FRAP and FLIP experiment simultaneously. It requires much less laser power, and there is no risk of not knowing how much of the recovery pool has been depleted. PhoC can also be used as a pulse chase to track the mobility and turnover of specific sub-populations of proteins in cells, without the need for blocking translation or prolonged photobleaching.

Using PhoC is also an opportunity to make more precise quantitative statements about protein mobility inside living cells. In interpreting FRAP and FLIP measurements, it is often assumed that proteins undergo Brownian motion in the cell. Based on this assumption, the measurements are fitted to a simple model to extract a diffusion coefficient (LippincottSchwartz and Patterson 2003). Since PhoC contains much more information than FRAP and FLIP (one time series for each location in the cell, rather than one time series per cell), PhoC measurements enable the testing of more adapt models for protein motion in the cell.

Another effective method for dynamic imaging with low photo-toxicity is the commonly known fluorescence correlation spectroscopy (FCS) (Elson and Magde 1974). FCS measures the dissipation time for a fluctuation to return to its equilibrium state. The principle is similar to FRAP where we measure recovery time after photobleaching, but in FCS, we measure spontaneous fluctuation rather than recovery from stimuli. Spontaneous fluctuation occurs, for example, because of changes in the number of fluorescent proteins in a volume due to Brownian motion in the cell. In FCS, fluorescence intensity fluctuation as a function of time is measured at a single or spot array, and an autocorrelation function of the measurements is calculated and fitted to a physical model of choice. Variations of this technique have developed over recent years. Image correlation spectroscopy (ICS), for example, uses a laser scanning microscope to calculate a 2D spatial autocorrelation function (Petersen et al. 1993). The correlation in ICS can be calculated over spatial and temporal dimensions, and the information collection rate is greater than in FCS, allowing for measurement time to be reduced dramatically. Furthermore, FCS, unlike ICS, acquires data from a relatively small region in the cell, which can be problematic since the cell is not homogenous. ICS, therefore, allows for a more accurate statistical analysis in less time and from a larger volume of the cell. ICS is currently limited by scan speeds, which must be greater than the speed of the molecule under consideration, thus restricting the use of this method to relativity slow processes. Raster ICS overcomes this limitation by incorporating a time delay between pixels and rows of pixels in the image (Digman et al. 2005). Other correlation microscopy methods include tools for analyzing single particles in dense environments, such as particle ICS (PICS) (Semrau and Schmidt 2007; Kolin and Wiseman 2007), total internal reflection FCS (TIR-FCS) which uses exponentially decaying EM waves to excite a thin layer of a specimen (Hassler et al. 2005), and selective plane imaging microscopy FCS (SPIMFCS), which enables measurement with high time resolution in a 3D image field (Wohland et al. 2010).

Some benefits of correlation microscopy include the potential ability to measure particle number, particle diffusion, the particle oligomerization state, protein binding, and protein folding. This information, however, must be extracted from an autocorrelation function, which can make it difficult to distinguish between different processes within the cell. Unlike PhoC, correlation spectroscopy does not enable tracking a sub-population of proteins from point $\mathrm{A}$ to point $\mathrm{B}$ in the cell, but instead provides information about the average mobility of all the labeled proteins within each region of interest.

\section{Imaging protein interactions}

Measuring protein interactions can provide an even more nuanced view of droplet formation. A wide variety of methods have been developed to observe protein-protein interaction. Forster resonance energy transfer (FRET), for example, has traditionally been used as an interaction reporter. Another method, which has been gaining popularity, is bimolecular 

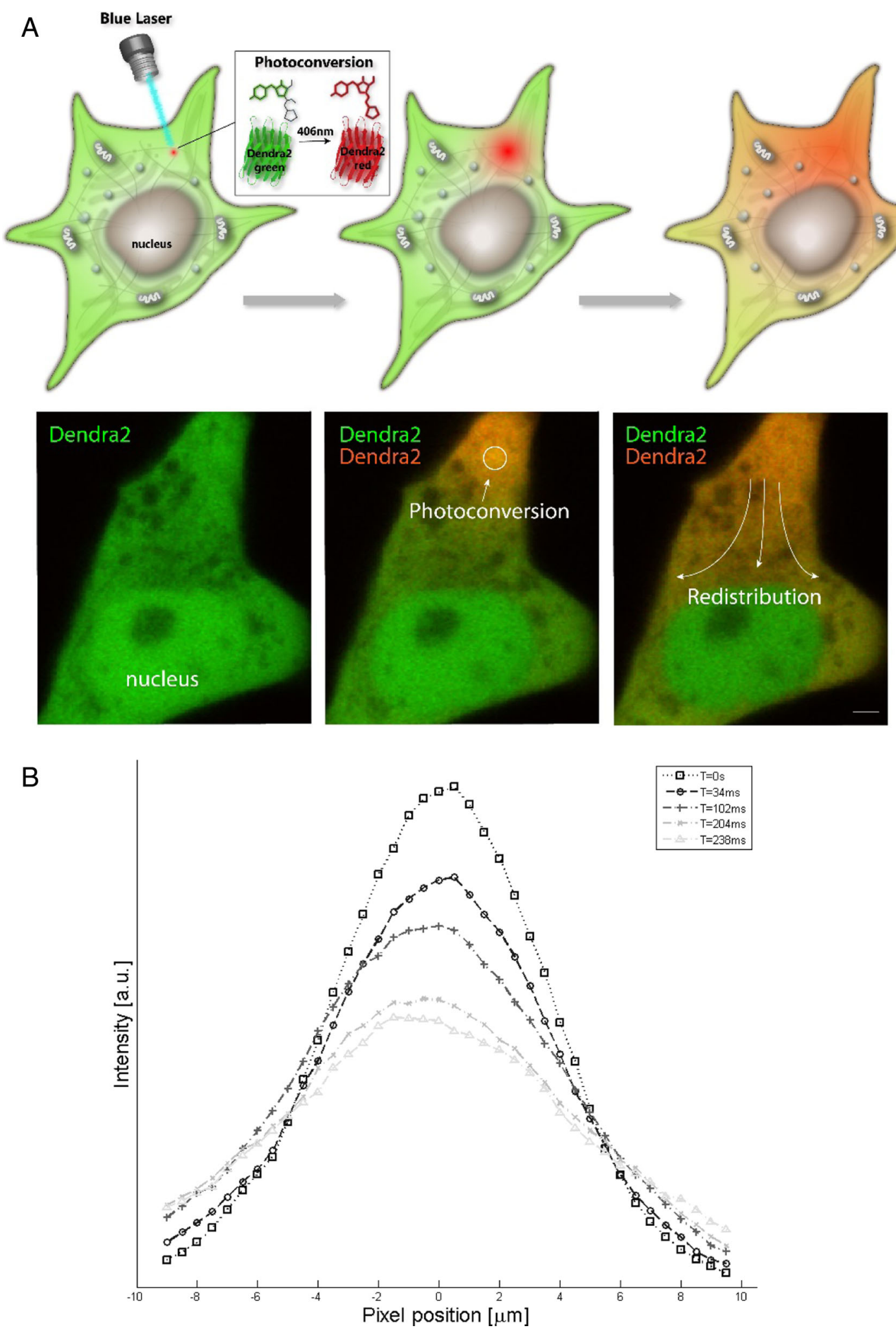

Fig. 1 a A schematic representation of Dendra2 photoconversion and the subsequent tracking of the fluorophore or fusion protein throughout the cell. b The average red fluorescence emission, measured in a line of pixels

fluorescence complementation (BiFC). BiFC enables direct visualization of protein interactions by a technique which perpendicular to the excitation laser beam, at various times. At $t=0$, photoactivation of cytoplasmic Dendra2 in cos-7 cells; at $t>0$, signal dissipation of the intensity profile can be measured

brings two non-fluorescing fragments of a fluorescent molecule into close proximity. BiFC was developed based on 
studies which show that cleaved enzymes that had lost their enzymatic ability can reacquire their enzymatic ability by positioning the fragments in close proximity to one another. Similarly, fragments of fluorescent proteins which are fused to a pair of interacting proteins can form a fluorescent protein complex (Hu et al. 2002; Kerppola 2006, 2008). One advantage of $\mathrm{BiFC}$ is that it can be used with weakly expressing genes of interest. In contrast, the quantum yield of FRET is inversely proportional to the sixth power of the distance between the interacting molecules (Piston and Kremers 2007), and therefore the amount of the interacting proteins must be high enough to notice small changes in illumination. BiFC also does not require a correction for other sources of fluorescence as does FRET. One drawback of BiFC is that the fluorophore fusion event is usually irreversible, essentially forming a permanent dimer from the interacting proteins. Hence, this method is ideal as an end-point analysis of interaction which is both well suited for live cells and relatively quantitative.

\section{Conclusion}

Over the past few years, better optics, more sensitive detectors, and innovations in scanning and SPIM microscopes, as well as the development of better fluorophores with desirable spectral and photoconversion properties, have enabled unprecedented levels of spatial and temporal resolution in live cell. As these techniques are applied to small dynamic compartments that are caused by and which manage the stress response, we stand to learn much more about how cells respond to changing conditions, if we look carefully.

Acknowledgments We thank Triana Amen for helpful discussion and help with generating figures and members of the Kaganovich lab for discussion and comments on the manuscript. We apologize to any colleagues if we unintentionally missed their studies or were unable to mention them due to space limitations. This work was supported by the European Research Council under the European Union's Seventh Framework Programme (FP/2007-2013)/ERC-StG2013 337713 DarkSide starting grant, an Israel Science Foundation Grant ISF 843/11, a German Israel Foundation Grant GIFI-1201-242.13/2012 (D.K.); an Israel Health Ministry grant under the framework of E-Rare-2, a Niedersachsen-Israel Research Program grant, and a grant from the Abisch-Frenkel Foundation.

\section{References}

Alberti S (2012) Molecular mechanisms of spatial protein quality control. Prion 6(5):437-442

Amen T, Kaganovich D (2014) Dynamic droplets: the role of cytoplasmic inclusions in stress, function, and disease. Cell Mol Life Sci 72(3): 401-415

Anderson P, Kedersha N (2002) Stressful initiations. J Cell Sci 115(16): $3227-3234$
Anderson P, Kedersha N (2006) RNA granules. J Cell Biol 172(6):803808

Ballatore C, Lee VM-Y, Trojanowski JQ (2007) Tau-mediated neurodegeneration in Alzheimer's disease and related disorders. Nat Rev Neurosci 8(9):663-672

Bashkirov VI et al (1997) A mouse cytoplasmic exoribonuclease (mXRN1p) with preference for G4 tetraplex substrates. J Cell Biol 136(4):761-773

Boisvert F-M et al (2007) The multifunctional nucleolus. Nat Rev Mol Cell Biol 8(7):574-585

Brangwynne CP (2013) Phase transitions and size scaling of membraneless organelles. J Cell Biol 203(6):875-881

Brangwynne CP et al (2009) Germline P granules are liquid droplets that localize by controlled dissolution/condensation. Science 324(5935): 1729-1732

Breker M, Gymrek M, Schuldiner M (2013) A novel single-cell screening platform reveals proteome plasticity during yeast stress responses. J Cell Biol 200(6):839-850

Brock KP et al. (2015) Structural basis for modulation of quality control fate in a marginally stable protein. Structure

Buchan JR, Parker R (2009) Eukaryotic stress granules: the ins and outs of translation. Mol Cell 36(6):932-941

Buchan JR et al (2013) Eukaryotic stress granules are cleared by autophagy and Cdc48/VCP function. Cell 153(7):1461-1474

Cherkasov Vet al (2013) Coordination of translational control and protein homeostasis during severe heat stress. Curr Biol 23(24):2452-2462

Couthouis J et al (2011) A yeast functional screen predicts new candidate ALS disease genes. Proc Natl Acad Sci 108(52):20881-20890

Decker $\mathrm{M}$ et al (2011) Limiting amounts of centrosome material set centrosome size in C. elegans embryos. Curr Biol 21(15):1259-1267

Digman MA et al (2005) Fluctuation correlation spectroscopy with a laser-scanning microscope: exploiting the hidden time structure. Biophys J 88(5):L33-L36

Eddy EM (1976) Germ plasm and the differentiation of the germ cell line. Int Rev Cytol 43:229-280

Ellis RJ (2001) Macromolecular crowding: obvious but underappreciated. Trends Biochem Sci 26(10):597-604

Elson EL, Magde D (1974) Fluorescence correlation spectroscopy. I. Conceptual basis and theory. Biopolymers 13(1):1-27

England JL, Kaganovich D (2011) Polyglutamine shows a urea-like affinity for unfolded cytosolic protein. FEBS Lett 585(2):381-384

Escusa-Toret S, Vonk WIM, Frydman J (2013) Spatial sequestration of misfolded proteins by a dynamic chaperone pathway enhances cellular fitness during stress. Nat Cell Biol 15(10):1231-1243

Filonov GS et al (2011) Bright and stable near-infrared fluorescent protein for in vivo imaging. Nat Biotechnol 29(8):757-761

Gall JG (2003) The centennial of the Cajal body. Nat Rev Mol Cell Biol 4(12):975-980

Gallina I et al (2015) Cmr1/WDR76 defines a nuclear genotoxic stress body linking genome integrity and protein quality control. Nat Commun 6:6533

Goehring NW, Hyman AA (2012) Organelle growth control through limiting pools of cytoplasmic components. Curr Biol 22(9):R330 R339

Gönczy P (2012) Towards a molecular architecture of centriole assembly. Nat Rev Mol Cell Biol 13(7):425-435

Grob A, Colleran C, McStay B (2014) Construction of synthetic nucleoli in human cells reveals how a major functional nuclear domain is formed and propagated through cell division. Genes Dev 28(3):220 230

Han TW et al (2012) Cell-free formation of RNA granules: bound RNAs identify features and components of cellular assemblies. Cell 149(4): 768-779

Hassler K et al (2005) Total internal reflection fluorescence correlation spectroscopy (TIR-FCS) with low background and high count-rate per molecule. Opt Express 13(19):7415-7423 
Hu C-D, Chinenov Y, Kerppola TK (2002) Visualization of interactions among bZIP and Rel family proteins in living cells using bimolecular fluorescence complementation. Mol Cell 9(4):789-798

Huisken J et al (2004) Optical sectioning deep inside live embryos by selective plane illumination microscopy. Science 305(5686):10071009

Hyman AA, Weber CA, Jülicher F (2014) Liquid-liquid phase separation in biology. Annu Rev Cell Dev Biol 30:39-58

Jucker M, Walker LC (2013) Self-propagation of pathogenic protein aggregates in neurodegenerative diseases. Nature 501(7465):45-51

Kaganovich D, Kopito R, Frydman J (2008) Misfolded proteins partition between two distinct quality control compartments. Nature 454(7208):1088-1095

Kato M et al (2012) Cell-free formation of RNA granules: low complexity sequence domains form dynamic fibers within hydrogels. Cell 149(4):753-767

Kedersha $\mathrm{N}$ et al (2005) Stress granules and processing bodies are dynamically linked sites of mRNP remodeling. J Cell Biol 169(6): 871-884

Kerppola TK (2006) Design and implementation of bimolecular fluorescence complementation (BiFC) assays for the visualization of protein interactions in living cells. Nat Protoc 1(3):1278-1286

Kerppola TK (2008) Bimolecular fluorescence complementation (BiFC) analysis as a probe of protein interactions in living cells. Annu Rev Biophys 37:465

King OD, Gitler AD, Shorter J (2012) The tip of the iceberg: RNAbinding proteins with prion-like domains in neurodegenerative disease. Brain Res 1462:61-80

Knowles TPJ, Vendruscolo M, Dobson CM (2014) The amyloid state and its association with protein misfolding diseases. Nat Rev Mol Cell Biol 15(6):384-396

Kolin DL, Wiseman PW (2007) Advances in image correlation spectroscopy: measuring number densities, aggregation states, and dynamics of fluorescently labeled macromolecules in cells. Cell Biochem Biophys 49(3): 141-164

Kremers G-J et al (2009) Photoconversion in orange and red fluorescent proteins. Nat Methods 6(5):355-358

Lagier-Tourenne C, Cleveland DW (2009) Rethinking ALS: the FUS about TDP-43. Cell 136(6):1001-1004

Li P et al (2012) Phase transitions in the assembly of multivalent signalling proteins. Nature 483(7389):336-340

Li YR et al (2013) Stress granules as crucibles of ALS pathogenesis. J Cell Biol 201(3):361-372

Lippincott-Schwartz J, Patterson GH (2003) Development and use of fluorescent protein markers in living cells. Science 300(5616):87-91

Luby-Phelps K (1999) Cytoarchitecture and physical properties of cytoplasm: volume, viscosity, diffusion, intracellular surface area. Int Rev Cytol 192:189-221

Mahen R, Venkitaraman AR (2012) Pattern formation in centrosome assembly. Curr Opin Cell Biol 24(1):14-23

Malinovska L et al (2012) Molecular chaperones and stress-inducible protein-sorting factors coordinate the spatiotemporal distribution of protein aggregates. Mol Biol Cell 23(16):3041-3056

Malinovska L, Kroschwald S, Alberti S (2013) Protein disorder, prion propensities, and self-organizing macromolecular collectives. Biochim Biophys Acta Proteins Proteomics 1834(5):918-931
Moldavski O et al (2015) Lipid droplets are essential for efficient clearance of cytosolic inclusion bodies. Dev Cell. doi:10.1016/j.devcel. 2015.04.015

Nguyen T, Bensaude O (1994) Increased thermal aggregation of proteins in ATP-depleted mammalian cells. Eur J Biochem 220(1):239-246

Ogrodnik M et al (2014) Dynamic JUNQ inclusion bodies are asymmetrically inherited in mammalian cell lines through the asymmetric partitioning of vimentin. Proc Natl Acad Sci 111(22):8049-8054

Pattabiraman S, Kaganovich D (2014) Imperfect asymmetry: the mechanism governing asymmetric partitioning of damaged cellular components during mitosis. Bioarchitecture just-accepted : 00-00

Petersen NO, Höddelius PL, Wiseman PW, Seger O, Magnusson KE (1993) Quantitation of membrane receptor distributions by image correlation spectroscopy: concept and application. Biophys J 65(3): $1135-1146$

Piston DW, Kremers G-J (2007) Fluorescent protein FRET: the good, the bad and the ugly. Trends Biochem Sci 32(9):407-414

Ross CA, Poirier MA (2004) Protein aggregation and neurodegenerative disease. S10-S17

Ross CA, Poirier MA (2005) What is the role of protein aggregation in neurodegeneration? Nat Rev Mol Cell Biol 6(11):891-898

Santi PA (2011) Light sheet fluorescence microscopy: a review. J Histochem Cytochem 59(2):129-138

Semrau S, Schmidt T (2007) Particle image correlation spectroscopy (PICS): retrieving nanometer-scale correlations from high-density single-molecule position data. Biophys J 92(2):613-621

Shaner NC et al (2008) Improving the photostability of bright monomeric orange and red fluorescent proteins. Nat Methods 5(6):545-551

Shevtsov SP, Dundr M (2011) Nucleation of nuclear bodies by RNA. Nat Cell Biol 13(2):167-173

Singer AB, Gall JG (2011) An inducible nuclear body in the Drosophila germinal vesicle. Nucleus 2(5):403-409

Spokoini R et al (2012) Confinement to organelle-associated inclusion structures mediates asymmetric inheritance of aggregated protein in budding yeast. Cell Rep 2(4):738-747

Udan M, Baloh RH (2011) Implications of the prion-related Q/N domains in TDP-43 and FUS. Prion 5(1):1-5

van den Berg B, John Ellis R, Dobson CM (1999) Effects of macromolecular crowding on protein folding and aggregation. EMBO J 18(24):6927-6933

Weber SC, Brangwynne CP (2012) Getting RNA and protein in phase. Cell 149(6):1188-1191

Webster AJ, Cates ME (1998) Stabilization of emulsions by trapped species. Langmuir 14(8):2068-2079

Weisberg SJ et al (2012) Compartmentalization of superoxide dismutase 1 (SOD1G93A) aggregates determines their toxicity. Proc Natl Acad Sci 109(39):15811-15816

Wohland T et al (2010) Single plane illumination fluorescence correlation spectroscopy (SPIM-FCS) probes inhomogeneous threedimensional environments. Opt Express 18(10):10627-10641

Zhou H-X, Rivas G, Minton AP (2008) Macromolecular crowding and confinement: biochemical, biophysical, and potential physiological consequences. Annu Rev Biophys 37:375

Zwicker D et al (2014) Centrosomes are autocatalytic droplets of pericentriolar material organized by centrioles. Proc Natl Acad Sci 111(26):E2636-E2645 